\title{
Management of the main postoperative surgical complications after transanal endoscopic microsurgery: an observational study
}

\author{
Xavier Serra-Aracil, Laura Mora-López, Anna Pallisera-Lloveras, Sheila Serra-Pla, Albert Garcia-Nalda, \\ Esther Gil-Barrionuevo, Salvador Navarro-Soto
}

Colorectal Surgery Unit, Department of General and Digestive Surgery, Parc Taulí University Hospital, Universitat Autònoma de Barcelona (UAB), Sabadell 08208, Barcelona, Spain.

Correspondence to: Dr. Xavier Serra-Aracil, Colorectal Surgery Unit, Department of General and Digestive Surgery, Parc Taulí University Hospital, Universitat Autònoma de Barcelona (UAB), Parc Taulí s/n, Sabadell 08208, Barcelona, Spain.

E-mail:xserraa@gmail.com

\begin{abstract}
How to cite this article: Serra-Aracil X, Mora-López L, Pallisera-Lloveras A, Serra-Pla S, Garcia-Nalda A, Gil-Barrionuevo E, Navarro-Soto S. Management of the main postoperative surgical complications after transanal endoscopic microsurgery: an observational study. Mini-invasive Surg 2019;3:37. http://dx.doi.org/10.20517/2574-1225.2019.36
\end{abstract}

Received: 13 Sep 2019 First Decision: 12 Nov 2019 Revised: 14 Nov 2019 Accepted: 3 Dec 2019 Published: 12 Dec 2019

Science Editor: Giulio Belli Copy Editor: Cai-Hong Wang Production Editor: Jing Yu

\begin{abstract}
Aim: Rates of clinically relevant postoperative morbidity after transanal endoscopic microsurgery (TEM) are low. For this reason, there are few descriptions in the literature on the management of these complications. Because of this lack of information, their importance may be either underestimated or overestimated (in the latter case, leading to overtreatment). The present article reports the frequency of the occurrence of postoperative surgical complications after TEM and describes various approaches to their management.
\end{abstract}

Methods: An observational study was carried out with prospective data collection and retrospective analysis from June 2004 to June 2019, including all patients undergoing TEM for rectal tumors. All postoperative complications were recorded using the Clavien-Dindo classification (CI-D), as well as preoperative, surgical, postoperative, and pathological variables.

Results: During the study period, 778 patients underwent TEM, of whom 716 met the inclusion criteria. Postoperative morbidity was $22.1 \%$ (158/716). Clinically relevant morbidity (CI-D > II) was 5\% (36/716). The most frequent complication was rectal bleeding, occurring in 115/716 (16.1\%) patients; 85 of these 115 (73.9\%) patients were grade I Cl-D. Urinary complications were rare (30/716, 4.2\%). Similarly, infectious complications of perianal and pelvic abscesses appeared in 7/716 (1\%) patients, two of whom required colostomy.

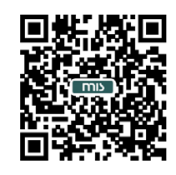


Conclusion: Clinically relevant complications after TEM are rare. For this reason, experience of these complications is limited. Here, we propose a management protocol to ensure that these complications are neither underestimated nor subjected to excessively aggressive or unnecessary treatment.

Keywords: Transanal endoscopic microsurgery, TEM, transanal endoscopic operation, minimally invasive surgery, morbidity and morbidity management

\section{INTRODUCTION}

The approach to benign or initially malignant rectal lesions through local surgery posed a considerable challenge until the advent of Transanal Endoscopic Microsurgery (TEM), introduced by Buess in the $1980 \mathrm{~s}^{[1]}$. Thanks to the creation of a pneumorectum, this technique makes it possible to perform local resections even beyond the rectum-sigmoid junction.

Later technical variations on TEM include transanal endoscopic operation $(\mathrm{TEO})^{[2]}$, which uses a high definition monitor, and TAMIS ${ }^{[3]}$ (TransAnal Minimally Invasive Surgery), a more recent development that incorporates a single-port system. The application of strict selection criteria and careful surgical techniques obtain good results for postoperative morbidity and mortality, function, and cure.

Overall postoperative morbidity after TEM ranges from $7.7 \%$ to $31.4 \%^{[4,5]}$. However, the absence of standardization in the recording and the description of the complications makes the results of different studies difficult to compare. A previous study by our group ${ }^{[6]}$ reported a morbidity rate of $23.6 \%$, grouped according to the Clavien-Dindo classification (Cl-D $)^{[7]}$. More than half of these complications (Cl-D grade I) required observation alone, and clinically relevant morbidity (Cl-D $\geq \mathrm{II})$ was recorded in only $5.6 \%$ of the patients.

The most frequent complications after TEM are rectal bleeding (as in the study just mentioned) ${ }^{[6]}$ or urinary morbidity, with reported rates ranging between $5.9 \%$ and $10.8 \%{ }^{[8,9]}$.

The management of complications after TEM has not been widely reported. Rectal bleeding, the most frequent complication, has a Cl-D classification ranging from I to IVa/b. In the remaining postoperative complications, such as urinary morbidity, infection, asymptomatic postoperative fever, and massive pneumo-retroperitoneum on computed tomography (CT) or chest radiography, it is unclear what protocol should be applied. The main aim of the present study was to describe the frequency of occurrence of postoperative surgical complications after TEM according to their Cl-D classification. The secondary aim was to describe the therapeutic management protocol in the most frequent complications.

\section{METHODS}

\section{Study design}

An observational, single-center study in consecutive patients undergoing TEM was carried out with prospective data collection and retrospective analysis. Computerized data management was carried out with the Microsoft Access 2003 software in a protected format.

\section{Patients and setting}

All patients were operated on by surgeons at the Parc Tauli University Hospital, Coloproctology Unit from June 2004 to June 2019. All patients with indication of TEM underwent a preoperative study protocol $^{[10]}$ incorporating endorectal ultrasound (US) and rectal magnetic resonance imaging (MRI). These examinations classify the patients into five groups of preoperative indication: Group I with curative 
intention (benign tumors), which, after US and MRI, are staged US-MRI,To-1 and US-MRI,No; Group II, with curative intent (low grade adenocarcinomas, US-MRI,To-1 and US-MRI,No); Group III, consensus indication (low grade adenocarcinomas, US-MRI,T2 and US-MRI,No) who reject radical surgery; Group IV, palliative care; and Group V, atypical indication ${ }^{[1]}$.

Inclusion criteria were patients in preoperative indication Groups I-IV who were candidates for TEM surgery.

Exclusion criteria were patients in preoperative indication Group V, and those who, after intraoperative assessment of possible TEM, were assigned to abdominal surgery on technical grounds.

\section{Preoperative preparation, surgical technique, and postoperative evolution}

In our protocol ${ }^{[10]}$, all patients with indication for TEM undergo anterograde mechanical preparation of the colon together with antibiotic and thromboembolic prophylaxis. General anesthetic is applied in most cases, unless the anesthesiologist decides to use spinal anesthesia due to the patient's condition. The techniques used for local rectal excision are either TEM (Richard Wolf, Knittlingen, Germany) or TEO (Karl Storz GmbH, Tüttlingen, Germany). Full wall resection is performed by ultrasound scalpel, following the superficial plane of the perirectal fat.

The lesion's defect on the rectal wall should be sutured to prevent complications due to stenosis of the rectal lumen (in large defects) and postoperative bleeding due to fecal erosions. A long-lasting 3-0 absorbable monofilament suture such as polydioxanone (PDS, MonoPlus) is used with a 20-22 gauge curved cylindrical atraumatic needle. A $10 \mathrm{~cm}$ length is cut to facilitate handling in the interior of the rectoscope. A Vicryl (Ethicon) clip is placed at the ends, using an instrument known as Lapra-TY for placement, as an anchor and to avoid knot tying. A curved needle holder is used, which facilitates handling the suture.

The suture should always be made in a transverse direction to avoid compromising the rectal lumen and to avoid formation of stenosis. The stitches are placed as full-thickness continuous sutures that are passed through the rectal wall, as previously described. Upon completion of suturing, irrigate once again with povidone iodine solution diluted to $1 \%$ with physiological saline solution.

The bladder catheter is removed at the end of the surgery. Oral diet and ambulation are initiated after $6 \mathrm{~h}$, and patients are discharged after $24 \mathrm{~h}$ unless they present complications.

\section{Main variable}

The main variable was post-surgical complications in patients undergoing TEO/TEM within 30 days of the intervention.

\section{Secondary variables}

The secondary variables were epidemiological, preoperative (patient- and tumor-dependent variables), surgical, postoperative (Clavien-Dindo Classification, Table 1) ${ }^{[7]}$, and pathological variables.

In addition, postoperative morbidity (postoperative complications or adverse effects), defined as an unexpected consequence or injury caused to the patient due to the treatment, not due to their underlying disease, was evaluated. Morbidity was considered clinically relevant with a Cl-D grade $\geq$ II requiring specific medical action.

Rectal bleeding was defined as more than $100 \mathrm{~mL}$ of red blood, or a bowel movement completely of blood (evaluated by nurse or surgeon). The same criteria were applied to patients consulting the emergency department. 
Table 1. Clavien-Dindo classification ${ }^{[7]}$

\begin{tabular}{|c|c|}
\hline Grade & Definition \\
\hline Grade I & $\begin{array}{l}\text { Any deviation from the normal postoperative course without the need for pharmacological treatment or surgical, } \\
\text { endoscopic, and radiological interventions } \\
\text { Allowed therapeutic regimens are: drugs as antiemetics, antipyretics, analgetics, diuretics, electrolytes, and } \\
\text { physiotherapy. This grade also includes wound infections opened at the bedside }\end{array}$ \\
\hline Grade II & $\begin{array}{l}\text { Requiring pharmacological treatment with drugs other than such allowed for grade I complications } \\
\text { Blood transfusions and total parenteral nutrition are also included }\end{array}$ \\
\hline \multirow[t]{3}{*}{ Grade III } & Requiring surgical, endoscopic or radiological intervention \\
\hline & Intervention not under general anesthesia \\
\hline & Intervention under general anesthesia \\
\hline Grade IV & Life-threatening complication (including CNS complications) ${ }^{\star}$ requiring IC/ICU management \\
\hline Grade IVa & Single organ dysfunction (including dialysis) \\
\hline Grade IVb & Multiorgan dysfunction \\
\hline Grade V & Death of a patient \\
\hline Suffix "d" & $\begin{array}{l}\text { If the patient suffers from a complication at the time of discharge (see examples in Table } 2 \text { ), the suffix " } d \text { " (for } \\
\text { "disability") is added to the respective grade of complication. This label indicates the need for a follow-up to fully } \\
\text { evaluate the complication }\end{array}$ \\
\hline
\end{tabular}

*Brain hemorrhage, ischemic stroke, subarrachnoidal bleeding, but excluding transient ischemic attacks. CNS: central nervous system; IC: intermediate care; ICU: intensive care unit

Since January 2005, morbidity has been prospectively recorded in all patients admitted to the Colorectal Unit and the Department of General and Digestive Surgery at our hospital ${ }^{[6]}$. The assessment of adverse effects is peer-reviewed. The present study was approved by the local Institutional Ethics Committee (CEIC: 2016-636) and complied with the criteria of the Declaration of Helsinki. The STROBE guidelines for observational studies were followed.

\section{Statistical analysis}

SPSS version 23 was used for statistical analysis. Prospective data collection allowed analysis of the data without the presence of missing values. The quantitative variables were described using mean values and standard deviation if normality criteria were met; otherwise, median, interquartile range (IQR), and range (R) were used. Categorical variables were described in absolute values and percentages.

\section{RESULTS}

During the study period, 788 patients underwent TEM in our Coloproctology Unit. Seventy-two patients did not meet the inclusion criteria, leaving a total of 716 patients. Figure 1 shows the patients included according to indication group.

Table 2 displays the epidemiological and preoperative variables of patients undergoing TEM. Median age was 71 years, and $430(60 \%)$ patients were men. Median lesion size was $4 \mathrm{~cm}$. Neoadjuvant treatment was administered in $44(6.1 \%)$ patients. Median distance from the lower edge of the lesion to the anal verge was $7 \mathrm{~cm}$, and from the upper edge to the anal verge was $11 \mathrm{~cm}$. The most frequent location was the lateral quadrant, reported in $318(44.4 \%)$ patients. Sessile morphology was the most common in 329 (47.1\%) patients.

As regards surgical, postoperative, and pathological variables [Table 3], 655 (91.4\%) patients underwent general anesthetic. The frequency of TEM and TEO use was similar (349 (48.7\%) and 367 (51.3\%), respectively), although in recent years TEO has been more widely used. En bloc resection was possible in 658 (91.9\%) patients. Median surgical time was $70 \mathrm{~min}$. Peritoneal cavity perforation was recorded in 51 (7.1\%) cases, without major morbidity and only one case required conversion to abdominal surgery. Vaginal perforation was observed in 12 patients; despite repair, five recto-vaginal fistulas appeared (5/12, 41.7\%). The overall postoperative morbidity rate was $22.1 \%$ (158/716), although 98 (13.7\%) complications were Cl-D 


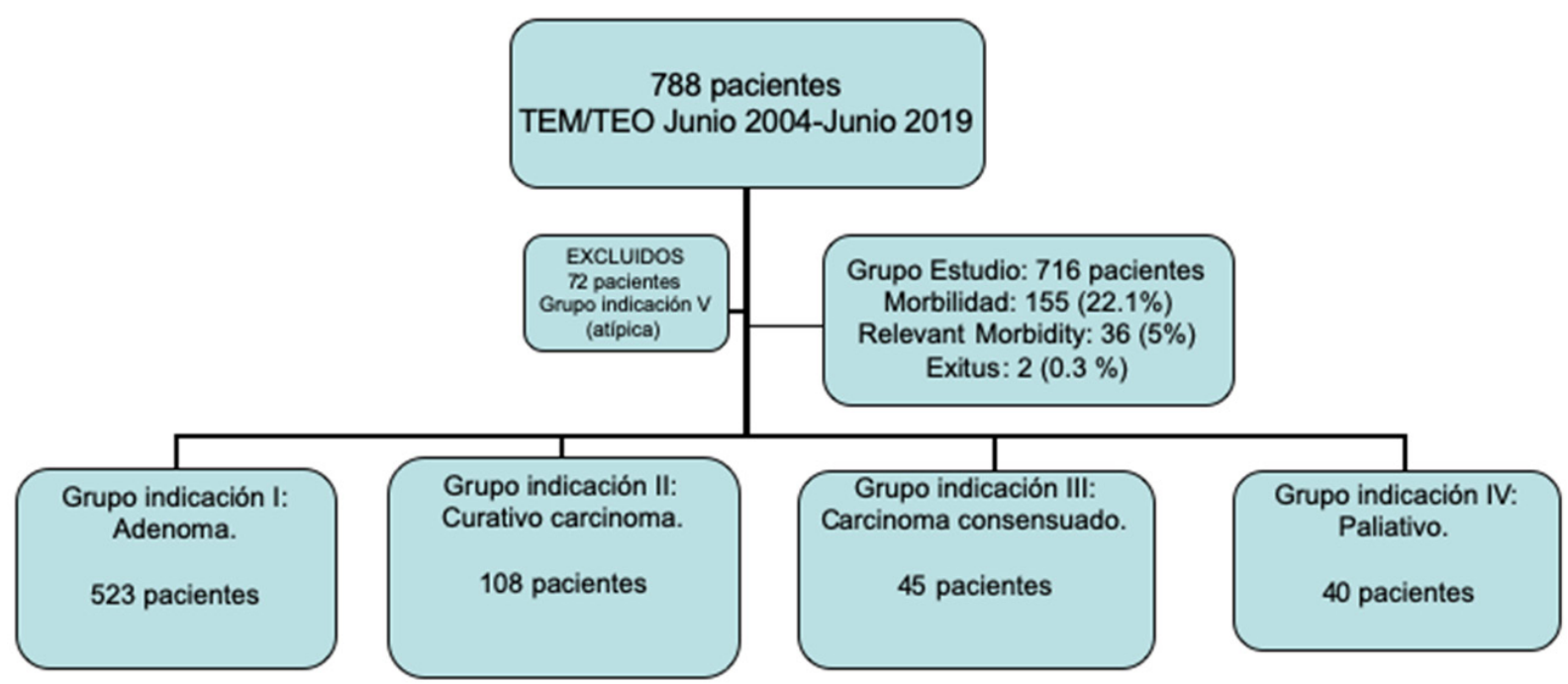

Figure 1. Flow chart of the study patients. TEM: transanal endoscopic microsurgery; TEO: transanal endoscopic operation

Table 2. Descriptive epidemiological and preoperative data of patients who underwent TEM

\begin{tabular}{|c|c|c|c|}
\hline Variables & & & $\begin{array}{c}\text { Overall patients } \\
n=716(\%)\end{array}$ \\
\hline \multirow[t]{20}{*}{ Epidemiology } & Age (years) (median-IQR-range) & & 71 (IQR 16) (range 31-92) \\
\hline & \multirow[t]{2}{*}{$\operatorname{Sex}(\%)$} & Male & $430(60 \%)$ \\
\hline & & Female & $286(40 \%)$ \\
\hline & Tumor size (cm) (median-IQR-range) & & 4 (IQR 2) (range 0.5-12) \\
\hline & Preoperative chemo-radiotherapy & & $44(6.1 \%)$ \\
\hline & Re-TEM & & $24(3.4 \%)$ \\
\hline & TEM after polypectomy with positive margin resection & & $48(6.8 \%)$ \\
\hline & Distance from anal verge $(\mathrm{cm})$ (median-IQR-range) & & 7 (IQR 5) (range 1-22) \\
\hline & Distance from tumor proximal margin to anal verge $(\mathrm{cm})$ (median-IQR-range) & & 11 (IQR 4.5) (range 1-26) \\
\hline & \multirow[t]{3}{*}{ Location of the tumor (\%) } & Anterior & $185(25.8 \%)$ \\
\hline & & Lateral & $318(44.4 \%)$ \\
\hline & & Posterior & $213(29.7 \%)$ \\
\hline & \multirow[t]{4}{*}{ Morphology of the lesion (\%) } & Flat & $169(23.6)$ \\
\hline & & Pedunculated & $150(21.3 \%)$ \\
\hline & & Sessile & $329(47.1 \%)$ \\
\hline & & Ulcerated & $54(7.6 \%)$ \\
\hline & \multirow[t]{4}{*}{ ASA (\%) } & I & $23(3.2 \%)$ \\
\hline & & II & $381(53.2 \%)$ \\
\hline & & III & $254(35.5 \%)$ \\
\hline & & IV & $58(8.1 \%)$ \\
\hline
\end{tabular}

TEM: transanal endoscopic surgery; ASA: American Society of Anesthesiology scale; IQR: Interquartile range; Re-TEM: recurrence-TEM

grade I. Clinically relevant morbidity (Cl-D $\geq$ II) was reported in $36(5 \%)$ patients with a Comprehensive Complication Index $(\mathrm{CCI})^{[12]}$ of 0 . Fifty-nine $(8.2 \%)$ patients presented asymptomatic postoperative fever. The causes of death in the two $(0.3 \%)$ patients who were exituswere reported in our previous publication ${ }^{[6]}$. The most frequent pathology was adenoma in 422 (58.9\%) patients. Full wall resection was achieved in 710 (99.2\%) patients and only $61(8.6 \%)$ patients presented positive margins.

Table 4 displays the most frequent types of complications related to TEM. In 139/158 (88\%) patients, the complications were surgical. The most frequent complication was rectal bleeding in 115/716 (16.1\%) patients; however, the bleeding was Cl-D grade I in 85/115 (73.9\%) patients. Fifteen of 115 (13\%) patients required surgical treatment (a new TEM in all cases). Rectal bleeding was a clinically relevant complication (Cl-D $\geq$ II) in $25 / 716(3.5 \%)$ patients. 
Table 3. Descriptive surgical, postoperative and pathological variables

\begin{tabular}{|c|c|c|c|}
\hline Variables & & & $\begin{array}{c}\text { Overall patients } \\
n=716\end{array}$ \\
\hline \multirow[t]{13}{*}{ Surgical } & \multirow[t]{2}{*}{ Anesthesia type } & General & $655(91.4 \%)$ \\
\hline & & Locoregional & $61(8.6 \%)$ \\
\hline & \multirow[t]{2}{*}{ Surgical equipment } & TEM & $349(48.7 \%)$ \\
\hline & & TEO & $367(51.3 \%)$ \\
\hline & \multirow[t]{2}{*}{ Fragmentation of the specimen } & En bloc & $658(91.9 \%)$ \\
\hline & & Piecemeal & $58(8.1 \%)$ \\
\hline & \multicolumn{2}{|l|}{ Surgical time(min) (median-IQR-range) } & 70 (IQR 50) (range 17-265) \\
\hline & \multicolumn{2}{|l|}{ Perforation into abdominal cavity } & $51(7.1 \%)$ \\
\hline & \multicolumn{2}{|l|}{ Vaginal perforation } & $12(1.7 \%)$ \\
\hline & \multirow[t]{3}{*}{ Suture of the defect after excision } & Complete & $614(85.8 \%)$ \\
\hline & & Incomplete & $94(13.1)$ \\
\hline & & Absent & $8(1.1 \%)$ \\
\hline & Conversion to abdominal surgery & & $1(0.1 \%)$ \\
\hline \multirow[t]{12}{*}{ Postoperative } & Overall morbidity & & $158(22.1 \%)$ \\
\hline & \multirow[t]{8}{*}{ Morbidity (Clavien-Dindo) } & 0 & $558(77.9 \%)$ \\
\hline & & । & $98(13.7 \%)$ \\
\hline & & $\|$ & $24(3.4 \%)$ \\
\hline & & IIla & $11(1.5 \%)$ \\
\hline & & IIIb & $17(2.4 \%)$ \\
\hline & & Iva & $5(0.7 \%)$ \\
\hline & & $\mathrm{IVb}$ & $1(0.1 \%)$ \\
\hline & & V (mortality) & $2(0.3 \%)$ \\
\hline & \multicolumn{2}{|l|}{ Clinically relevant morbidity $(\mathrm{Cl}-\mathrm{D}>\mathrm{II})$} & $36(5 \%)$ \\
\hline & \multicolumn{2}{|l|}{$\mathrm{CCl}$} & O (IQR 0) (range 0-100) \\
\hline & \multicolumn{2}{|l|}{ Asymptomatic fever post-TEM } & $59(8.2 \%)$ \\
\hline \multirow[t]{6}{*}{ Pathology } & \multirow[t]{3}{*}{ Definitive pathology } & Adenoma & $422(58.9 \%)$ \\
\hline & & Adenocarcinoma & $239(33.4 \%)$ \\
\hline & & No pathology & $55(7.7 \%)$ \\
\hline & Positive margin & & $61(8.6 \%)$ \\
\hline & \multirow[t]{2}{*}{ Wall excision } & Full-thickness & $710(99.2 \%)$ \\
\hline & & Partial & $6(0.8 \%)$ \\
\hline
\end{tabular}

TEM: transanal endoscopic microsurgery; TEO: transanal endoscopic operation; IQR: interquartile range; Cl-D: Clavien-Dindo; CCI: comprehensive complex index

Urinary complications were relatively uncommon, being recorded in 30/716 (4.2\%) patients. Nine of 716 (1.3\%) patients presented urine infections and $20(2.8 \%)$ patients presented acute urine retention (AUR).

Infectious complications were rare (14/716, 2\%). Pelvic or perianal abscess occurred in seven (1\%) patients and were treated by antibiotics and local debridement, except in two cases that required colostomy. All of them were associated with tumors located in the lower third of the rectum. In the cases that required colostomy, one was an immunosuppressed patient with lymphoma who developed perineal cellulitis. In the other patient, with no history of interest, a perianal abscess was observed on Postoperative Days 4-5; after local debridement, the perianal infection progressed, obliging the creation of a colostomy.

Two patients underwent exploratory laparotomy, one for severe pneumoperitoneum on chest X-ray [Figure 2], and the other due to massive neuro-retroperitoneum on abdominal CT [Figure 3]. The abdominal CT did not record any free intra-abdominal fluid or collections. In neither case was rectal perforation observed, or the presence of intestinal contents.

\section{DISCUSSION}

Postoperative surgical complications after TEM are rare and tend to be unimportant. In this study, 98/158 (62.1\%) complications recorded were Cl-D grade I, and only 5\% of patients presented clinically relevant 


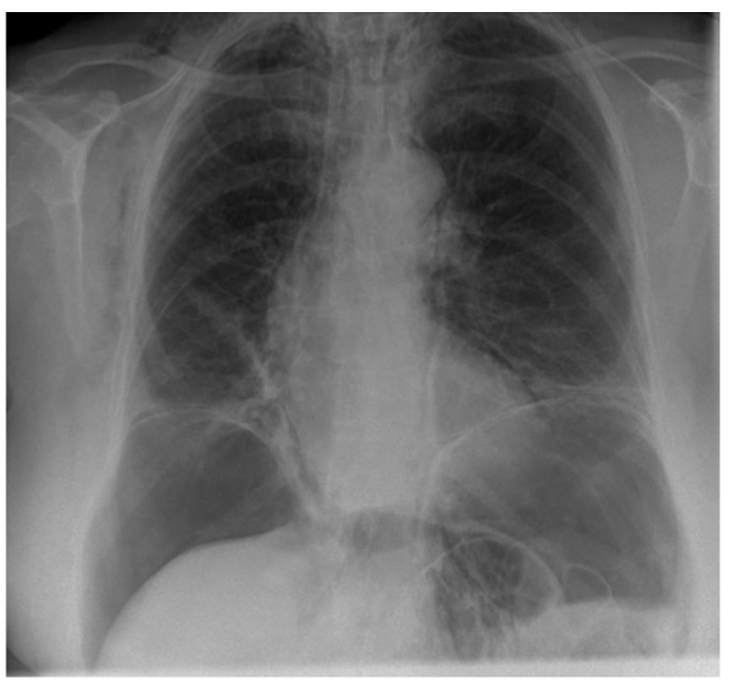

Figure 2. Chest X-ray with massive pneumoperitoneum

Table 4. Description of surgical complications related to TEM

\begin{tabular}{|c|c|c|c|}
\hline \multicolumn{3}{|c|}{ Total number of patients with complications related to TEM } & 158/716 (22.1\%) \\
\hline \multicolumn{3}{|c|}{ Patients with surgical complications } & $139(19.4 \%)$ \\
\hline \multicolumn{3}{|c|}{ Patients with medical complications } & $13(1.8 \%)$ \\
\hline \multicolumn{3}{|c|}{ Patients with both medical and surgical complications } & $6(0.8 \%)$ \\
\hline \multirow[t]{9}{*}{ Rectal bleeding } & Overall morbidity & & $115 / 716(16.1 \%)$ \\
\hline & Morbidity (Cl-D) & I & $85 / 115(73.9 \%)$ \\
\hline & & $\|$ & $5 / 115(4.3 \%)$ \\
\hline & & IIla & $10 / 115(8.7 \%)$ \\
\hline & & IIIb & $11 / 115(9.6 \%)$ \\
\hline & & IVa & $4 / 115(3.5 \%)$ \\
\hline & & $\mathrm{IVb}$ & $0 / 115(0 \%)$ \\
\hline & & V (mortality) & $0 / 115(0 \%)$ \\
\hline & Clinically relevant morbidity $(\mathrm{Cl}-\mathrm{D}>\mathrm{II})$ & & $25 / 716(3.5 \%)$ \\
\hline \multicolumn{3}{|c|}{ Urinary complications } & $30 / 716(4.2 \%)$ \\
\hline \multicolumn{3}{|c|}{ AUR } & $20 / 716(2.8 \%)$ \\
\hline \multicolumn{3}{|c|}{ UTI } & $9 / 716(1.3 \%)$ \\
\hline \multicolumn{3}{|c|}{ Hematuria and traumatic urine catheter insertion } & $5 / 716(0.7 \%)$ \\
\hline \multicolumn{3}{|c|}{ Infectious complications } & $14 / 716(2 \%)$ \\
\hline \multicolumn{3}{|c|}{ Abscess } & $7 / 716(1 \%)$ \\
\hline \multicolumn{3}{|c|}{ Pneumoperitoneum/retropneumoperitoneum/pneumomediastinum } & $2 / 716(0.3 \%)$ \\
\hline \multicolumn{3}{|c|}{ Recto-vaginal fistula } & $5 / 716(0.7 \%)$ \\
\hline
\end{tabular}

TEM: transanal endoscopic microsurgery; AUR: acute urine retention; UTI: urinary tract infection; CI-D: Clavien-Dindo

complications (Cl-D $\geq \mathrm{II})$. We believe that the description of postoperative complications should apply the same classification to allow comparison of the results. The Clavien-Dindo classification ${ }^{[7]}$ is probably the most widely used in the literature; the Comprehensive Complication Index (CCI ${ }^{[12]}$ is useful for completing the description of complications in procedures associated with high morbidity, but in TEM it is of little value $^{[6]}$.

As noted above, there are few references to the management of these complications in the literature. Because of the lack of information, some of these complications may be overtreated; alternatively, others may be ignored and may evolve into more complex forms. For this reason, the objective of this study was to assess the most frequent types of complication based on our experience and on the literature. 

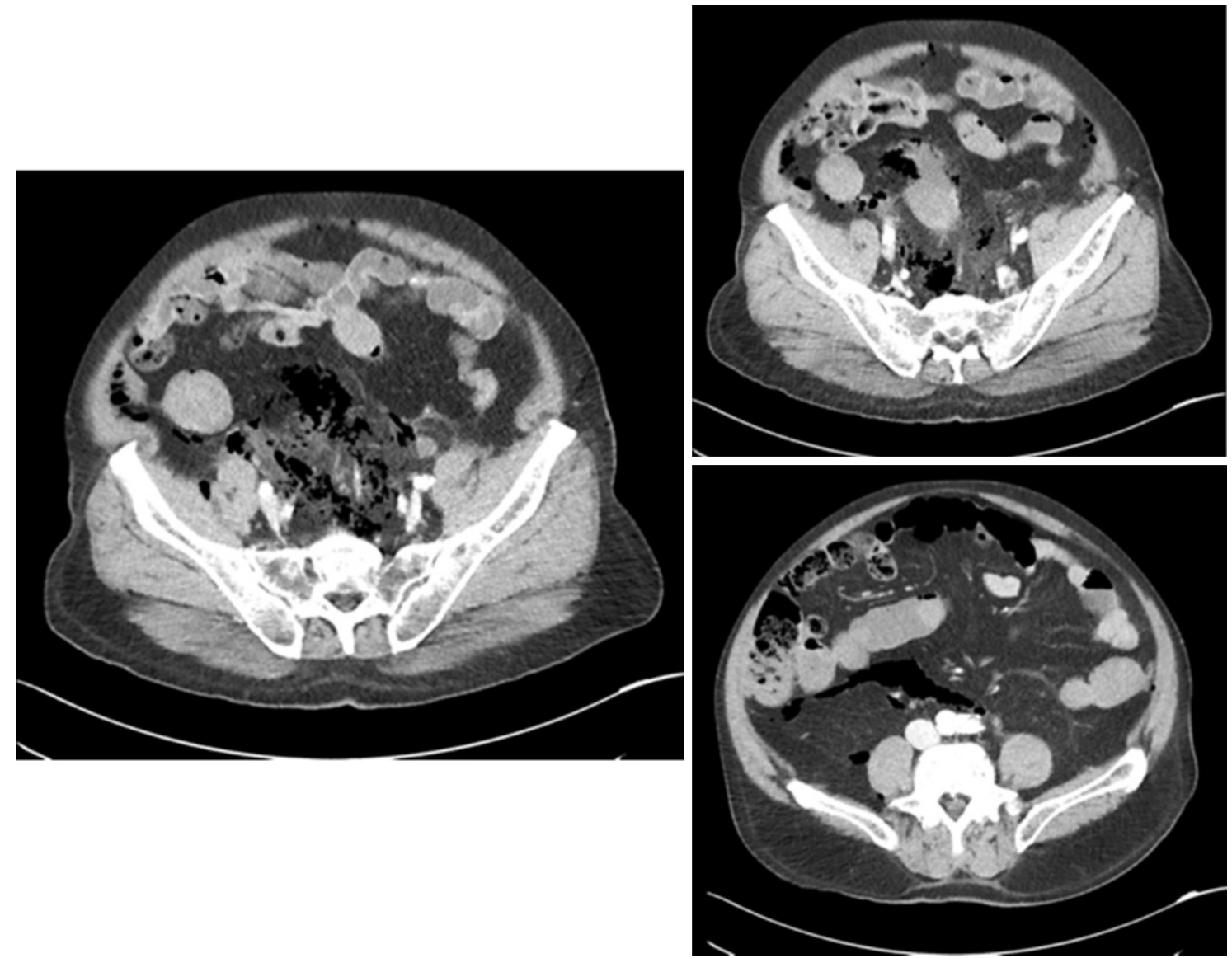

Figure 3. Abdominal computed tomography scan with pneumoretroperitoneum, without intra-abdominal free fluid or collections

\section{Rectal bleeding}

The presence of a wound inside the rectum, whether completely sutured, partially sutured, or left open, causes a minimal blood emission after contact with the feces and the internal pressure of the rectum with defecation. This is a common occurrence and is considered normal within the process. Therefore, it is important to define the concept of rectal bleeding ${ }^{[6]}$.

Table 4 shows that rectal bleeding is the most frequent complication, accounting for $72.8 \%$ (115/158) of the total complications. Eighty-four of these 115 (73\%) complications were Cl-D grade I. For this reason, when a patient consults our hospital's emergency department or after the immediate postoperative period, the first approach is conservative: hemodynamic control, withdrawal of anticoagulant or antiplatelet medication, and observation. If the complication persists, with a decrease in hemoglobin $(>2-3 \mathrm{~g} / \mathrm{dL})$ or hemodynamic alteration, the next step is to perform a flexible rectosigmoidoscopy under sedation in an attempt to visualize the source of bleeding and perform sclerosis or insert a hemostatic clip. In our study, this maneuver was performed in 25 patients with clinically relevant morbidity (Cl-D $\geq \mathrm{II})$ and was effective in 10 cases $(40 \%)$.

The rest of the patients operated upon for rectal bleeding $(15 / 115,13 \%)$ were controlled with a new TEM. None underwent abdominal surgery or arteriography embolization. However, the performance of a new TEM requires a number of precautionary steps. Bleeding may be due to a deshiscence suture, or may be observed directly over the resection bed if it has not been sutured. First, as the rectal lumen is filled completely with blood clots, we will not be able to see anything inside via the rectoscope. The first step 
is to introduce the TEM rectoscope into the rectum without the working attachment. Then, the rectum undergoes intensive washing to remove all the blood clots from the rectal ampulla. Subsequently, the working attachment is introduced once more and the rectum is distended. In many cases, a rectal catheter is inserted via the TEM to complete the aspiration of the blood clots, since the conventional TEM aspirator is not effective. The bleeding may be as low diffuse bleeding or may start from a particular point; the amounts are small, but constant. The inflammation of the tissues means that any sutures inserted would tear. The most effective procedure is to perform coagulation with aspiration maintained over the point of bleeding and the entire surface of the resection bed. Finally, new washes are performed and the defect is left open.

\section{Urinary complications}

Some studies have reported urinary complications to be the most frequent, affecting between $5.9 \%$ and $10.8 \%$ of patients ${ }^{[8,9]}$. In our study, 30/716 (4.2\%) patients presented urinary morbidity and only $20(2.8 \%)$ patients had AUR, a complication which other studies (e.g., Kumar et al..$^{[8]}$ ) have reported to be more frequent. One possible explanation is that in our protocol we remove the bladder catheter at the end of the surgery in all patients except those with a history of prostate disease; in these latter patients, the medication against benign prostatic hyperplasia is not withdrawn and the catheter is removed early the next morning. Another possible reason is the use of general anesthetic in 655/716 (91.4\%) patients, which does not favor AUR (unlike spinal anesthetic).

\section{Peritoneal cavity perforation}

Peritoneal cavity perforation has been considered a cause of major morbidity in some studies ${ }^{[13]}$; others ${ }^{[14,15]}$, however, have not found it to be a significant risk factor for postoperative complications. In our view, if peritoneal perforation is detected intraoperatively and is repaired by TEO/TEM, this is considered as a standard technical variant of the procedure ${ }^{[15]}$.

\section{Anterior resections with perforation in the vagina and recto-vaginal fistulas. Urethral lesions?}

Special care must be taken in the resection of anterior lesions in women. The integrity of the recto-vaginal septum should be monitored, and a vaginal examination performed in case of doubt. Vaginal perforation should be considered an important complication; indeed, five of our 12 patients with vaginal perforations developed recto-vaginal fistula, due to the poor vascularization of the recto-vaginal septum and the pressure exerted on it during defecation. These fistulas constitute a rare complication (appearing in five of our $716(0.7 \%)$ patients), but they are difficult to treat. In most cases, they require reoperation with a temporary stoma and subsequent repair of the fistula. We have registered no urethral injuries after TEM in our experience.

\section{Postoperative pneumo-retropneumoperitoneum}

As shown in Figures 2 and 3, the appearance of massive pneumo- and retroperitoneum on chest radiographs and abdominal $\mathrm{CT}$ is relatively common during the immediate postoperative period. Provided that the clinical and inflammatory parameters are normal, pneumo- and retroperitoneum need not be a matter for concern. As noted above, two patients with asymptomatic postoperative fever presented these features and were assigned to exploratory laparotomy, which turned out to be negative in both cases.

\section{Asymptomatic postoperative fever}

Fever is defined as asymptomatic and postoperative if it appears during the first $24-48 \mathrm{~h}$, without other symptoms, hemodynamic repercussion, or any focus. Fever may be as high as $39^{\circ} \mathrm{C}^{[6]}$; it is not associated with leukocytosis or with abdominal or pelvic pain and remits with antipyretics. In our study, 59/716 (8.2\%) patients presented fever, which we considered to be a normal feature of the postoperative course. In contrast, fever associated with abdominal pain and leukocytosis is termed post-TEM syndrome ${ }^{[16]}$. 
The etiology of this asymptomatic fever is not known, although it is probably caused by an inflammatory reaction to the surgical aggression rather than to a self-limited infection or contamination; in all cases, it was controlled with antipyretics. We do not consider it as postoperative complication.

\section{Severe perianal infection and the need for colostomy}

The removal of lesions in the rectum, with fecal contamination of the resection area, entails a high risk of developing serious and frequent pelvic infections. Infections are very rare (in our study, only seven (1\%) cases were recorded) but they can be serious. When they appear, antibiotic treatment and local debridement should be applied as quickly as possible to avoid the need for more radical treatments. Two of these patients required an end colostomy: one an immunosuppressed patient with a lymphoma, and the other without associated morbidity.

The limitations of this study are those inherent in observational studies performed at a single center. Its main strength is the unusually large sample size. All the data reported were recorded prospectively over a period of 15 years; the experience gathered over this time has allowed us to discuss and establish protocols for responding to the different complications.

In conclusion, clinically relevant complications after TEM are rare occurrences. Nonetheless, a protocol for their management needs to be established to ensure that their importance is not underestimated, and to avoid unnecessary or excessively aggressive treatments.

\section{DECLARATIONS}

\section{Acknowledgments}

We thank the rest of the members of the Coloproctology Unit for applying the study protocol. We thank Cristina Gomez Vigo for correcting the manuscript and Michael Maudsley for helping with the English.

\section{Authors' contributions}

Made substantial contributions to conception and design of the study and performed data analysis and interpretation: Serra-Aracil X, Mora-López L, Pallisera-Lloveras A, Serra-Pla S

Performed data acquisition and provided administrative, technical, and material support: Garcia-Nalda A, Gil-Barrionuevo E, Navarro-Soto S

\section{Availability of data and materials}

Not applicable.

\section{Financial support and sponsorship}

None.

\section{Conflicts of interest}

All authors declared that there are no conflicts of interest.

\section{Ethical approval and consent to participate}

The present study was approved by the Local Institutional Ethics Committee (CEIC: 2016-636), and complied with the criteria of the Declaration of Helsinki. The STROBE guidelines for observational studies were followed.

\section{Consent for publication}

Not applicable. 


\section{Copyright}

(c) The Author(s) 2019.

\section{REFERENCES}

1. Buess G, Hutterer F, Theiss J, Böbel M, Isselhard W, et al. A system for a transanal endoscopic rectum operation. Chirurg 1984;55:67780.

2. Rocha JJ, Feres O. Transanal endoscopic operation: a new proposal. Acta Cir Bras 2008;23:93-104; Discussion 104.

3. Atallah S, Albert M, Larach S. Transanal minimally invasive surgery: a giant leap forward. Surg Endosc. 2010;24:2200-5.

4. Allaix ME, Arezzo A, Caldart M, Festa F, Morino M. Transanal endoscopic microsurgery for rectal neoplasms: experience of 300 consecutive cases. Dis Colon Rectum 2009;52:1831-6.

5. Laliberte AS, Lebrun A, Drolet S, Bouchard P, Bouchard A. Transanal endoscopic microsurgery as an outpatient procedure is feasible and safe. Surg Endosc. 2015;29:3454-9.

6. Serra-Aracil X, Labró-Ciurans M, Rebasa-Cladera P, Mora-López L, Pallisera-Lloveras A, et al. Morbidity after transanal endoscopic surgery. Risk factors for postoperative complications in a one-day surgery program. Surg Endosc 2019;33:1508-17.

7. Dindo D, Demartines N, Clavien PA. Classification of surgical complications: a new proposal with evaluation in a cohort of 6336 patients and results of a survey. Ann Surg 2004;240:205-13.

8. Kumar AS, Coralic J, Kelleher DC, Sidani S, Kolli K, et al. Complications of transanal endoscopic microsurgery are rare and minor: a single institution's analysis and comparison to existing data. Dis Colon Rectum 2013;56:295-300.

9. Tsai BM, Finne CO, Nordenstam JF, Christoforidis D, Madoff RD, et al. Transanal endoscopic microsurgery resection of rectal tumors: outcomes and recommendations. Dis Colon Rectum 2010;53:16-23.

10. Serra-Aracil X, Mora-Lopez L, Alcantara-Moral M, Caro-Tarrago A, Gomez-Diaz CJ, et al. Transanal endoscopic surgery in rectal cancer. World J Gastroenterol 2014;20:11538-45.

11. Serra-Aracil X, Mora-Lopez L, Alcantara-Moral M, Corredera-Cantarin C, Gomez-Diaz C, et al. Atypical indications for transanal endoscòpic microsurgery to avoid major surgery. Tech Coloproctol 2014;18:157-64.

12. Slankamenac K, Graf R, Barkun J, Puhan MA, Clavien PA. The comprehensive complication index: a novel continuous scale to measure surgical morbidity. Ann Surg 2013;258:1-7.

13. Palma P, Freudenberg S, Samel S, Post S. Transanal endoscopic microsurgery: indications and results after 100 cases. Colorectal Dis 2004;6:350-5.

14. Gavagan JA, Whiteford MH, Swanstrom LL. Full-thickness intraperitoneal excision by transanal endoscopic microsurgery does not increase short-term complications. Am J Surg 2004;187:630-4.

15. Serra-Aracil X, Pallisera-Lloveras A, Mora-Lopez L, Rebasa P, Serra-Pla S, et al. Perforation in the peritoneal cavity during transanal endoscopic microsurgery for rectal tumors: a real surgical complication with a challenging prognosis? Surg Endosc 2019;33:1870-9.

16. Brown C, Raval MJ, Phang PT, Karimuddin AA. The surgical defect after transanal endoscopic microsurgery: open versus closed management. Surg Endosc 2017;31:1078-82. 